\title{
Responses of Gastrointestinal Peptides and Motor Activity to Milk and Water Feedings in Preterm and Term Infants
}

\author{
CAROL LYNN BERSETH, CYNTHIA K. NORDYKE, MARIA G. VALDES, BARBARA L. FURLOW, \\ AND VAY LIANG W. GO \\ Department of Pediatrics and Gastroenterology Research Unit, Mayo Clinic and Foundation, \\ Rochester, Minnesota 55905
}

\begin{abstract}
Because duodenal motor activity differs between preterm and term infants during fasting, this study evaluated the responses of motor activity and peptide release in response to feeding. In the first study, fasting concentrations of gastrin, gastric inhibitory peptide, neurotensin, and peptide YY (PYY) were determined in 53 preterm and 20 term infants. Plasma concentrations of gastrin and neurotensin were significantly lower in preterm infants than in healthy adults reported previously by our lab $(p<0.01)$. Plasma concentration of gastric inhibitory peptide and PYY were higher than in healthy adults $(p<$ $0.01)$. Gastrin concentrations in preterm and term infants varied directly with gestational age $(p<0.005)$; PYY varied inversely with gestational age $(p<0.005)$. In a secondary study, intestinal manometry was recorded and serial peptide concentrations were determined in 43 preterm babies who were given their first enteral feeding intraduodenally with formula or sterile water. Although none of the four peptide plasma concentrations changed in response to feeding with water, plasma concentrations of gastric inhibitory peptide, neurotensin, and PYY significantly increased with formula feedings ( $p<0.05$ or less). In addition, plasma gastrin increased significantly in seven infants fed milk compared with eight fed water by orogastric tube $(p<0.01)$. In contrast to the peptide response to feeding, motor activity changed in response to feeding with either water or milk; motility indices increased and periods of motor quiescence decreased significantly during feeding as compared with fasting $(p<0.02)$. Responses of both motor activity and peptides to feeding were time related. Although fasting concentrations of four regulatory peptides were immature in preterm infants compared with adults, postprandial responses to nutrient feedings were present in the first days of life. This discrepancy in functional maturation of the preterm intestine during fasting and feeding is present for both motor activity and peptide response, and we speculate that the controlling mechanisms of these two phases of digestion may mature independently at different postconceptual ages. (Pediatr Res 31: 587-590, 1992)
\end{abstract}

\section{Abbreviations}

GIP, gastric inhibitory peptide

NT, neurotensin

Received July 29, 1991; accepted February 3, 1992

Correspondence and reprint requests: C. L. Berseth, M.D., Department of Pediatrics, Division of Newborn Medicine, Baylor College of Medicine, One Baylor Plaza, Houston, TX 77030.

Supported by Grant HD24558 from the National Institutes of Health, Bethesda,
PYY, peptide YY

NICU, Newborn Intensive Care Unit

Motor activity, which is responsible for the aboral movement of nutrients through the gastrointestinal tract is modulated by neural and hormonal influences. We have recently shown that fasting intestinal motor activity differs between preterm and term infants (1) and, in turn, between infants and adults. Despite the presence of these differences in motor activity during fasting, the motor responses to feeding are similar in preterm and term infants (2). In a parallel fashion, fasting intestinal hormone concentrations differ between preterm infants and adults (3). However, hormonal responses to feeding in preterm and term infants are present, although blunted or overly reactive, in the 1 st postnatal wk when cross-sectional sampling techniques are used $(3,4)$. No previous study in preterm infants has validated that hormonal responses to feeding are present in neonates by using serial sampling of plasma concentrations of peptides. Inasmuch as motor responses to enteral feedings are present in preterm infants, we hypothesized that peptide responses to feedings would also be present. The purpose of the preliminary study was to determine plasma concentration of four gastrointestinal hormones and peptides in preterm and term infants and to assess the influence of gestational maturation on their concentration. The purpose of the secondary study was to compare serial peptide concentrations and motor activity in infants fed water or artificial formula to determine if peptide concentrations change in response to enteral feeding.

\section{SUBJECTS AND METHODS}

First study: levels of peptides during fasting. Study partıcipants in this first study were 63 preterm and 20 term infants admitted to Saint Mary's Hospital NICU. All infants were admitted for oxygen or ventilator support for respiratory disease, including respiratory distress syndrome, congenital pneumonia, or meconium aspiration. All infants had indwelling central venous or arterial catheters for routine NICU support and monitoring. Preterm infants ranged from 25 to $35 \mathrm{wk}$ of gestational age, and term infants from 36 to $42 \mathrm{wk}$ (Table 1). Preterm infants participated in this study on postnatal d 3.8 and term infants on $d 4.1$ (Table 1). By NICU routine, all babies were receiving i.v. fluids and had not received any enteral feedings. This protocol was approved by the Mayo Clinic Institutional Review Board, and parents provided informed consent for their infants' participation in this study. A single 2-mL blood sample was withdrawn from each infant's indwelling central catheter. Blood was placed in tubes containing $1.5 \mathrm{mg}$ of EDTA per $\mathrm{mL}$ of whole blood. 
Table 1. Patient characteristics and comparison of fasting plasma gastrointestinal peptide concentrations in preterm and term infants and adults (mean $\pm S E M$ )

\begin{tabular}{lccc}
\hline & Preterm & Term & Adults* \\
\hline$n$ & 63 & 21 & 15 \\
Gestational age (wk) & $30.9 \pm 0.3$ & $37.7 \pm 0.4$ & $5.4 \pm 0.7$ \\
Postnatal age (d) & $4.2 \pm 0.2$ & $3167 \pm 189$ & \\
Birth weight $(\mathrm{g})$ & $1570 \pm 74$ & $82 \pm 9 \dagger \ddagger$ & $100 \pm 10 \dagger \ddagger$ \\
Peptide concentrations (ng/L) & $59 \pm 4 \dagger$ & $588 \pm 70 \dagger \S$ & $230 \pm 26 \dagger$ \\
Gastrin & $578 \pm 36 \dagger \S$ & $32 \pm 6 \dagger \S$ & $67 \pm 8 \dagger$ \\
GIP & $27 \pm 4 \dagger \S$ & $530 \pm 64 \dagger \S$ & $75 \pm 9 \dagger$ \\
NT & $748 \pm 64 \dagger \S$ & & \\
PYY & & & \\
\hline
\end{tabular}

* Previously reported in Ref. 5 .

$\dagger p<0.0001$, analysis of variance among age groupings.

$\ddagger$ Differ from preterms, Newman-Keuls test.

$\S$ Differ from aduits, Newman-Keuls test.

Samples were centrifuged, and the plasma was stored at $-20^{\circ} \mathrm{C}$. RIA was used to determine in each sample the concentration of gastrin, GIP, NT, and PYY. Duplicate $100-\mu \mathrm{L}$ samples were used for each assay for gastrin, NT, and PYY; $200-\mu \mathrm{L}$ samples were used for GIP. The characteristics of these assays have been previously published (5). In brief, all assays had an intraassay variation of $8 \%$ or less and all plasma concentrations of infants fell within the range of absolute sensitivity for each assay used.

Feeding study. In this secondary study, 58 additional preterm infants participated as subjects; 43 received duodenal feedings and another 15 received gastric feedings. Infants ranged from 27 to $33 \mathrm{wk}$ of gestational age and had been admitted for respiratory distress syndrome. All were receiving parenteral nutrition and had received no enteral feedings. All were studied by postnatal d 3 . This protocol was also approved by the Mayo Clinic Institutional Review Board, and parents provided informed consent for their infant's participation in this study. Each infant was studied on the day that enteral feedings were to be initiated. Motor activity was evaluated using a neonatal manometric system that has been previously described and validated (6). In short, this system is a low-compliance, continuous perfusion system that provides a response rate of $57 \mathrm{~mm} \mathrm{Hg} / \mathrm{s}$ at $10 \mathrm{psi}$ at a flow rate of $0.01 / \mathrm{mL} / \mathrm{min} /$ recording port (6). Before any feedings were given, a $3.5-\mathrm{mm}$ motility/feeding tube was placed in the upper duodenum and the position verified by the presence of motor activity characteristic of the duodenum. Motor activity was recorded for $4 \mathrm{~h}$. At the end of $4 \mathrm{~h}$, blood was collected for determination of regulatory peptide concentrations. Each of 43 infants was then randomly assigned to receive a feeding with sterile water or Similac formula intraduodenally via the motility/ feeding tube. All infants were fed $4 \mathrm{~mL} / \mathrm{kg}$ as a 2 -h continuous infusion. Blood samples of $1.2 \mathrm{~mL}$ were withdrawn from each infant's indwelling central catheter at 30,60,150, and $180 \mathrm{~min}$. Blood was processed and peptide concentrations determined as for the preliminary study. The additional 15 infants were fed 4 $\mathrm{mL} / \mathrm{kg}$ feeding of milk $(n=7)$ or water $(n=8)$ by orogastric tube, and serial sampling was performed as described for infants fed by oroduodenal tube.

Data analysis. In the first study, plasma concentrations of all four peptides were compared among preterm infants, term infants, and adults by analysis of varience, with Newman-Keuls testing to identify the presence of intergroup differences. Regression analysis was performed for each peptide to assess peptide concentration in infants as a function of gestational age. Significant differences were identified when $p$ was less than 0.05 . In the secondary study, plasma concentrations of peptides at $30-180$ min were determined, and the peptide release during feeding was quantified by determining an integrated area under the curve for each peptide using the trapazoid rule. These integrated areas were compared between water- and milk-fed infants by unpaired $t$ test. Data analysis for babies fed duodenally and intragastrically was performed separately and expressed as mean \pm SEM.
In the manometric study, motor activity was analyzed in $30-$ min segments. Quiescence was defined as an absence of motor activity. Duration of quiescence was expressed as min of quiescence/h of recording/recording lead. Motility index, a quantification to identify the presence of the fed response, was calculated as $\log _{c}$ (sum of amplitude $\times$ number of pressure waves +1$)(7)$. Quiescence duration and motility index were compared during fasting and feeding infusion by paired $t$ test. To further characterize the feeding response (2), the number of pressure waves $/ 30$ min were calculated for each 30-min interval throughout the 180-min study and each value was compared with that for time 0 by paired $t$ test.

\section{RESULTS}

Preliminary study of peptide levels during fasting. Fasting plasma concentrations of all four hormones and regulatory peptides differed among preterm infants, term infants, and healthy nonpregnant females previously reported by our laboratory (5), (Table 1; all $p<0.0001$ ). Gastrin concentrations were lower in preterm infants than in term infants and adults, whereas NT concentrations were lower in both preterm infants and term infants compared with adults. GIP and PYY concentrations were significantly higher in preterm and term infants than in adults. Gastrin concentrations varied directly with gestational age $(r=$ $0.33 ; p<0.005)$. GIP and NT did not vary with gestational age. PYY varied inversely with gestational age $(r=-0.38 ; p<0.001)$.

Peptide responses to feeding. There was no significant difference in plasma concentrations in gastrointestinal peptides during fasting in water and milk-fed infants (Table 2). Water- and milkfed infants differed with respect to their hormonal response to feeding. Plasma gastrin did not significantly change throughout the 3-h study period in either milk- or water-fed infants, and their integrated responses were similar (Fig. 1). In contrast to gastrin, the integrated response of GIP, NT, and PYY were significantly greater in milk-fed infants compared with water-fed infants (all $p<0.05$ or less, Fig. 1).

In contrast to the infants fed by intraduodenal infusion, gastrin

Table 2. Characteristics of preterm infants fed water or milk (mean \pm SEM)

\begin{tabular}{lccc}
\hline & Water & Milk & $p$ \\
\hline$n$ & 21 & 22 & \\
Gestational age (wk) & $30.6 \pm 0.5$ & $31.5 \pm 0.5$ & NS \\
Birth weight (g) & $1552 \pm 117$ & $1725 \pm 126$ & NS \\
Postnatal age (d) & $4.9 \pm 0.5$ & $5.3 \pm 0.5$ & NS \\
Fasting plasma concentration (ng/L) & & & \\
$\quad$ Gastrin & $59 \pm 6$ & $60 \pm 7$ & NS \\
$\quad$ GIP & $533 \pm 54$ & $641 \pm 59$ & NS \\
$\quad$ NT & $19 \pm 3$ & $31 \pm 7$ & NS \\
PYY & $793 \pm 100$ & $566 \pm 84$ & NS \\
\hline
\end{tabular}




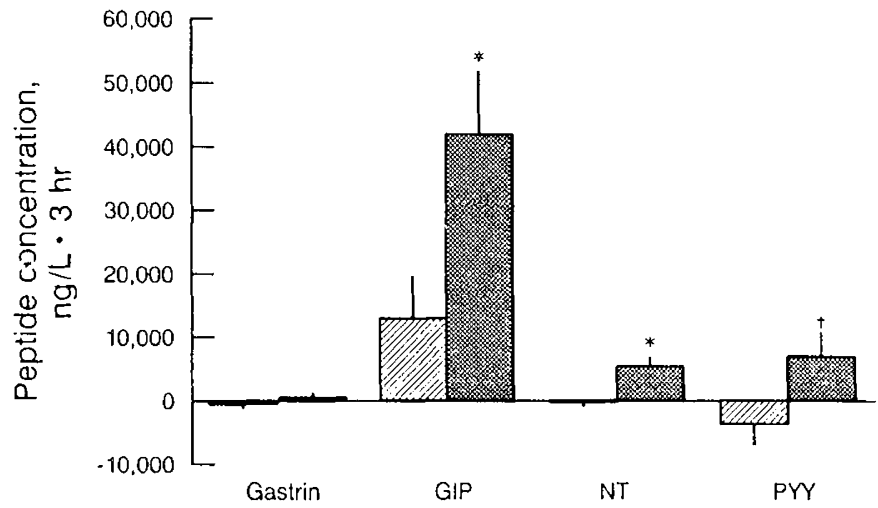

Fig. 1. Integrated concentrations of gastrointestinal peptides in preterm infants fed water or formula by oroduodenal tube (mean \pm SEM). Infants fed water are represented by bars with diagonal cross-hatching and infants fed formula, by stippled bars. Significant differences are designated by $*(p<0.05)$ and $\dagger(p<0.01)$.

Table 3. Motor activity during fasting and feeding in water and milk-fed preterm infants (mean \pm SEM)

\begin{tabular}{llcl}
\hline & Fasting & Fed & \multicolumn{1}{c}{$p$} \\
\hline Water-fed & & & \\
$\quad$ Motility index* & $12.6 \pm 0.2$ & $13.3 \pm 0.2$ & 0.001 \\
$\quad$ Quiescence duration (min/h/lead) & $22.7 \pm 1.8$ & $18.0 \pm 2.6$ & 0.02 \\
Milk-fed & & & \\
$\quad$ Motility index* & $12.6 \pm 0.2$ & $13.3 \pm 0.1$ & 0.001 \\
$\quad$ Quiescence duration (min/h/lead) & $23.5 \pm 1.8$ & $17.7 \pm 1.9$ & 0.02 \\
\hline
\end{tabular}

* Motility index, sum of $\log _{\mathrm{e}}$ (sum of amplitudes $x$ number of pressure waves +1 )

concentrations differed with milk or water feedings when infants were fed by orogastric tube. Plasma gastrin did not differ over $180 \mathrm{~min}$ in eight water-fed infants. In contrast to water-fed infants, plasma gastrin in seven milk-fed infants was significantly higher at 30 and $60 \mathrm{~min}$ compared with fasting, and the integrated gastrin response to milk feeding was significantly greater than that to water feeding $(137 \pm 100$ versus $1260 \pm 320 \mathrm{pg} /$ $\mathrm{mL} \cdot 1 \mathrm{~h} ; p<0.05)$.

Motor activity responses to feeding. In response to feeding, motor activity of the small intestine changed in both the milkfed and water-fed infants compared with fasting (Table 3). Motility index increased significantly in both groups during feeding compared with fasting (Table $3 ; p<0.001$ ). Conversely, quiescence decreased significantly during feeding compared with fasting (Table $3 ; p<0.02$ )

Timed responses of peptides and motor activity. Responses of peptides and motor activity to formula feeding were time-related. Gastrin concentration was significantly increased at 30 and 60 $\min (p<0.01)$ but decreased to values similar to fasting at 150 and $180 \mathrm{~min}$. GIP and PYY concentrations were significantly elevated at 30,60,120, and 150 min compared with fasting (all $p<0.01$ or less). NT was significantly higher at 60 and 120 min compared with fasting but declined at $180 \mathrm{~min}$ to values similar to fasting. Motor activity response to feeding, characterized by the number of peaks/30 min, also changed with time. The number of peaks/30 min significantly increased compared with fasting within $30 \mathrm{~min}$ of the initiation of the formula infusion; they remained elevated throughout the feeding infusion and declined to fasting values within $30 \mathrm{~min}$ after the feeding was completed (all $p<0.01$ ).

\section{DISCUSSION}

Plasma concentrations of gastrin, GIP, NT, and PYY during fasting were significantly different in preterm and term infants compared with healthy adult women. Gastrin and NT, potent stimulants of motility and peptide and acid release, were significantly lower in preterm infants compared with adults. GIP and PYY, potent inhibitors of motility and peptide secretion, were significantly higher in preterm infants compared with adults. Plasma concentrations of GIP and PYY were also significantly higher in term infants than healthy adults, suggesting that further postnatal maturation of these peptide concentrations occurs in infancy. These data concur with those showing cord blood PYY concentrations to be significantly higher in neonates than in healthy adults (8) and the presence of postnatal maturation of other peptides such as motilin and human pancreatic polypeptide (9).

All four of the peptides measured in this study are important modulators of lower esophageal sphincter tone, gastric emptying, and intestinal motility. Our previous studies have shown that small intestinal motility patterns differ in preterm infants and term infants. Furthermore, many characteristics of intestinal motor activity patterns in preterm infants "mature" with gestational age (1) and become more similar to those seen in term infants. This current study shows that gastrin and PYY significantly vary with gestational age, suggesting that there may be a temporal association of peptide concentrations and intestinal motility. Facer (10) has shown that fetal small intestine cell numbers of gastrin, GIP, and PYY increase with gestational age. We have recently shown that fasting gastrin concentration is significantly predictive of maturation of intestinal motility patterns in preterm infants (11). Therefore, plasma concentrations of these regionally distributed peptides may serve as markers in determining the functional maturation of preterm intestinal motor activity.

Both water-fed and milk-fed babies demonstrated a change in motor activity in response to enteral feedings. However, the calculation of motility index and quiescence were used to demonstrate only that a response to feeding had occurred. Although motor responses to feedings are nutrient-related in both infants (12) and adults (13), we did not characterize the motor activity responses to feeding sufficiently to determine if the responses in this study were nutrient related.

Water-fed infants demonstrated no change in plasma peptide concentration during the 180 -min study. Milk-fed infants experienced a significant rise in GIP, NT, and PYY. Although babies fed by oroduodenal tube demonstrated no increase in gastrin with feeding, gastrin increased significantly in babies fed by gastric tube, suggesting that oroduodenal feedings had failed to provide adequate stimulation to antral $G$ cells. Although $G$ cells are present in both neonatal antrum and duodenum, the maturation of antral $\mathrm{G}$ cells occurs postnatally in concert with maturation of plasma gastrin concentration (14). Collectively, these data suggest that antral $G$ cells play a more significant role than the duodenal cells in regulating neonatal gastrin response to nutrient stimulation.

The rise in peptide concentration in response to nutrients was time related. G cells are localized to the antrum and duodenum, and GIP is released in response to the presence of nutrient in the duodenum. NT is released in the jejunum and PYY in the distal ileum and colon in response to the presence of intraluminal nutrients. Therefore, the monitoring of serial peptides demonstrates indirectly that active aboral transit of intraluminal milk may have occurred. The presence of motor activity that migrates distally during fasting is thought to contribute significantly to the aboral movement of intraluminal nutrients. Many preterm infants lack this distally migrating activity. Despite this "immaturity" of preterm fasting intestinal motor activity, the postprandial rise in NT and PYY confirms that aboral movement of netrients occurred, suggesting that neonatal nonmigrating motor activity may in fact contribute to aboral transit.

Both motor activity and peptide concentrations in preterm infants are parallel in maturity. Fasting motor activity is immature in preterm infants compared with term infants, but motor activity changes in response to feeding (2). Similarly, fasting 
plasma peptide concentrations of preterm infants differ from those of adults, but peptide concentrations increase in response to feeding. The presence of this apparent functional maturity of the intestine in response to feeding suggests that the use of early enteral nutrition may be warranted in preterm infants, although the interval of feedings may need to be lengthened. The provision of early hypocaloric nutrition is associated with a rise in fasting gastrin and GIP (14). These fasting concentrations, in turn, are associated with improved fasting motor activity and clinical tolerance of routine feedings. We speculate that the monitoring of fasting motor activity and hormones are more relevant measurements of functional maturation of the preterm intestine than are feeding responses. However, the preterm intestine discerns nutrients from nonnutrients, and future studies should identify nutrients that enhance optimal functional responsiveness of the preterm intestine. These studies would provide important insights to design better feeding regimens for preterm infants.

\section{REFERENCES}

1. Berseth CL 1989 Gestational evolution of small intestinal motility in preterm and term infants. J Pediatr 115:646-651

2. Berseth CL 1990 Neonatal small intestinal motility: motor responses to feeding in term and preterm infants. J Pediatr 1 17:777-782

3. Lucas A, Bloom SR, Aynsley-Green A 1980 Development of gut hormone responses to feeding in neonates. Arch Dis Child 55:678-682
4. Gemelli M. Mami C, Manganaro R. Artemisia A, Nibali SC, Magazzu G Lombardo G 1987 Gastrin 17 and gastrin 34, before and after a meal in newborn infants. J Pediatr Gastroenterol Nutr 6:717-720

5. Berseth CL. Michener SR, Nordyke CK, Go VLW 1990 Postpartum changes in pattern of gastrointestinal regulatory peptides in human milk. Am J Clin Nutr 51:985-990

6. Amarnath RP, Berseth CL, Malagelada J-R, Perrault J, Abell TL, Hoffman AD 1989 Postnatal maturation of small intestinal motility in preterm infants. J Gastrointestinal Motility 1:138-143

7. Maladelada J-R, Camilleri M, Stanghellini V 1986 Manometric Diagnosis of Gastrointestinal Motility Disorders. Thieme, Inc. New York

8. Adrian TE, Smith HA, Calvert SA, Aynsley-Green A, Bloom SR 1986 Elevated plasma peptide $Y Y$ in human neonates and infants. Pediatr Res 20:12251227

9. Janik JS, Track NS, Filler RM 1982 Motilin, human pancreatic polypeptide, gastrin and insulin plasma concentrations in fasted children. $J$ Pediatr $101: 51-56$

10. Facer $\mathrm{P}$, Bishop AE, Cole GA, Altchison $\mathrm{M}$, Kendall $\mathrm{CH}$, van Aswegen $\mathrm{G}$ Penketh RJA, Rodek CH, McKeever P, Polak JM 1989 Developmental profile of chromogranin, hormonal peptides, and 5-hydroxytryptamine in gastrointestinal endocrine calls. Gastroenterology 97:48-57

11. Berseth CL, Go VLW 1990 Correlation of gastrointestinal hormones with maturation of neonatal small intestine motility. Gastroenterology 97:A178(abstr)

12. Koenig W, Berseth CL 1992 Manometrics for preterm infants: a new tool for old questions. Am J Perinatol (in press)

13. Takeuchi K. Peitsch W, Johnson L 1981 Mucosal gastrin receptor. V. Development in newborn rats. Am J Physiol 240:G163-G169

14. Berseth CL 1991 Early hypocaloric enteral feedings induce functional maturation of preterm intestine. Pediatr Res 29:29 1 A(abstr) 